\title{
Bounds on R-parity violating SUSY Yukawa couplings from semileptonic decays of baryons
}

\author{
Farida Tahir 4, M. Sadiq, M. Anwar Mughal, K. Ahmed \& \\ Department of Physics, Quaid-i-Azam University, Islamabad, Pakistan
}

\begin{abstract}
We consider tree-level corrections to hypercharge changing semileptonic decays of certain baryons induced by a minimal supersymmetric standard model with explicitly broken $R$-parity via $L$-violation. This study leads to a new set of constraints on the products of couplings arising from the $\mathrm{LQd}^{c}$ operator of the superpotential.
\end{abstract}

\footnotetext{
${ }^{1}$ E-mail: anwar@sgs.sdnpk.undp.org

${ }^{2}$ E-mail: kahmed@hep-qau.sdnpk.undp.org
} 
Lepton number $(L)$ is conserved in the Standard Model (SM) of electroweak interactions; discovery of $L$-violation would be recognized as a manifestation of physics beyond the SM. Among the possible extensions of the SM, supersymmetry (SUSY) in its minimal form known as the minimal supersymmetric SM (MSSM) could be the most attractive possibility of $L$ violation. In general, the MSSM could contain renormalizable $L$ - violating interactions as well as the baryon number $(B)$ violating interaction which are described by the superpotential [1]

$$
f^{\Delta L \neq 0}=\frac{1}{2} \lambda_{i j k}\left[\mathrm{~L}_{i} \mathrm{~L}_{j}\right] \mathrm{e}_{k}^{c}+\lambda_{i j k}^{\prime} \mathrm{L}_{i} \mathrm{Q}_{j} \mathrm{~d}_{k}^{c}+\lambda_{i j k}^{\prime \prime} \mathrm{u}_{i}^{c} \mathrm{~d}_{j}^{c} \mathrm{~d}_{k}^{c}
$$

where $i, j, k$ are generation indices, L, Q are the lepton and quark left-handed doublets and $e^{c}, u^{c}\left(d^{c}\right)$ are the charge conjugates of the right-handed lepton and up(down) quark singlets, respectively. $\lambda_{i j k}, \lambda_{i j k}^{\prime}$ and $\lambda_{i j k}^{\prime \prime}$ are the Yukawa couplings. $\lambda_{i j k}$ and $\lambda_{i j k}^{\prime \prime}$ are antisymmetric in $i, j$ and $j, k$ indices respectively. Renormalizable $L$ - and $B$-violating gauge invariant interactions are the characteristic features of the SUSY theory. The doubling of particle spectrum so arising leads to the introduction of squarks and sleptons which couple to standard fermions through $L$ - and $B$-violating couplings. There has been a lot of work in the recent years on the effects associated with the $R$-breaking couplings in (11). $R$ - breaking interactions contribute to various low-energy processes through the virtual exchange of sparticles which in turn put bounds on the $R$-breaking couplings. Among many papers constraining individual couplings include the constraints from atomic parity violation and 
$e D$-asymmetry, $\nu_{\mu}$ DIS [2], $\left(\nu_{0}\right)_{\beta \beta}$ decay [3], neutrino mass [4], decays of $K^{+}$, top quark [5] and $Z$ [6]. More recent reviews on the effects of $R$-breaking with updated bounds on the couplings are given in Ref. [7]. Phenomenologies of these couplings at various colliders have also been investigated recently [ [ 8 ]. Note that together, the couplings in the $L$ - and $B$-violating terms in (1)), lead to a rapid proton decay [9]. The standard remedy for this is enforcing a new symmetry called the $R$ - parity [ [,, 9$]$ which is defined as $R=(-)^{3 B+L+2 S}$, with a value +1 for ordinary particles and -1 for their SUSY partners. As an alternate a phenomenology of $R$-breaking due to explicit $L$-violation is equally viable that can also provide a natural protection to proton by disallowing $B$-violating interaction.

While establishing the $R$-violating operators in (1), it is generally assumed that one $R$-violating operator is dominant at a time. Under this assumption sometime stronger bounds on the products of $R$-violating operators are neglected. There are already some papers where bounds on the products $\lambda^{\prime} \lambda^{\prime}$ were derived from various processes. Some important bounds include a bound on the product $\lambda_{113}^{\prime} \lambda_{131}^{\prime}$ from the neutrinoless double beta decay [2], $\lambda_{i 13}^{\prime} \lambda_{i 31}^{\prime}$ and $\lambda_{i 12}^{\prime} \lambda_{121}^{\prime}$ from neutral mesons mixing, $\lambda_{1 j 1}^{\prime} \lambda_{2 j 1}^{\prime}, \lambda_{11 k}^{\prime} \lambda_{21 k}^{\prime}$ from muonium conversion, and $\lambda_{1 j 1}^{\prime} \lambda_{2 j 2}^{\prime}$ from the flavour changing decays of $K$ [11. The decays of $\tau$ into lepton and vector/pseudoscalar mesons puts constraints on $\lambda_{31 k}^{\prime} \lambda_{11 k}^{\prime}$ and $\lambda_{31 k}^{\prime} \lambda_{21 k}^{\prime}$ [12]. Recently new limits on various $\lambda^{\prime} \lambda^{\prime}$ products have been derived from the lepton-flavour violating $Z$-decays [13] and from the consideration of $\Delta S=2$ and $\Delta B=2$ box graphs [14]. 
In this note we consider the hypercharge changing semileptonic decays of baryons to derive a new set of bounds on certain products of $\lambda^{\prime}$ couplings by calculating the $L$ - violating contribution to such decays. The relevant Lagrangian in terms of component fields is given by

$$
L_{\lambda^{\prime}}=\lambda_{i j k}^{\prime}\left[\begin{array}{c}
\bar{d}_{k R} \nu_{i L} \widetilde{d}_{j L}+\bar{d}_{k R} d_{i L} \widetilde{\nu}_{j L}+\overline{\left(\nu_{i L}\right)^{c}} d_{j L} \widetilde{d}_{k R}^{*} \\
-\bar{d}_{k R} e_{i L} \widetilde{u}_{j L}-\bar{d}_{k R} u_{j L} \widetilde{e}_{i L}-\overline{\left(e_{i L}\right)^{c}} u_{j L} \widetilde{d}_{k R}^{*}
\end{array}\right]+\text { h.c. }
$$

Evidently, the above $L$-violating couplings manifest themselves only when the two non-spectator quarks form an $S U(2)_{L}$ doublets. To be explicit we consider a typical hypercharge changing semileptonic baryonic decay: $B \longrightarrow$ $B^{\prime} l \bar{\nu}_{l}(l=e, \mu)$ with a baryon in the final state. We have chosen $\Delta Y=1$ decay modes as they involve the products of $\mathrm{LQd}^{c}$ operators. All such decays can be represented by quark subprocess $s \longrightarrow u l \bar{\nu}$. These decays proceed in the SM by a tree-level $W$-exchange Feynman graph. In the presence of interaction (2) an additional squark- exchange diagram contributes to these decays. The current structure of this new interaction is given by

$$
L_{e f f}=-\frac{\lambda_{i 1 k}^{\prime} \lambda_{i 2 k}^{\prime}}{m_{\widetilde{d_{k}}}^{2}}\left[\overline{\left(l_{i L}\right)^{c}} u_{L} \overline{s_{L}}\left(\nu_{i L}\right)^{c}\right] .
$$

Thus, the amplitude for the process induced by (3) is given by

$$
M_{\not R}=\frac{\lambda_{i 1 k}^{\prime} \lambda_{i 2 k}^{\prime}}{m_{\widetilde{d_{k}}}^{2}} \bar{l}\left(1+\gamma_{5}\right) u \bar{s}\left(1-\gamma_{5}\right) \nu_{l} .
$$

After Fierz's re-ordering (丑) takes the form

$$
M_{\not R}=\frac{\lambda_{i 1 k}^{\prime} \lambda_{i 2 k}^{\prime}}{m_{\widetilde{d_{k}}}^{2}} \bar{\nu}_{l} \gamma_{\sigma}\left(1-\gamma_{5}\right) l \bar{s} \gamma^{\sigma}\left(1-\gamma_{5}\right) u
$$


Adding (5) in SM's contribution, the total amplitude can be expressed in the form

$$
M^{\prime}=\left(\frac{G^{\prime}}{\sqrt{ } 2}\right) \bar{\nu}_{l} \gamma_{\sigma}\left(1-\gamma_{5}\right) l \bar{s} \gamma^{\sigma}\left(1-\gamma_{5}\right) u
$$

where $G^{\prime} / \sqrt{ } 2=\left(G_{F} V_{u s} / \sqrt{ } 2+\lambda_{i 1 k}^{\prime} \lambda_{i 2 k}^{\prime} / 8 m_{\widetilde{d}_{k}}^{2}\right)$. In the limit of vanishing lepton masses the branching ratio for a baryonic decay under consideration is given by

$$
\begin{aligned}
\operatorname{Br}\left(B \longrightarrow B^{\prime} l \bar{\nu}_{l}\right)= & \tau_{B} \frac{G^{\prime 2} g_{V}^{2}(0)}{60 \pi^{3}}\left(\frac{m_{B^{\prime}}}{m_{B}}\right)^{3 / 2} \Delta^{5} \times \\
& {\left[1+3 \alpha^{2}+\left(\frac{\Delta^{2}}{2 m_{B} m_{B^{\prime}}}\right)(0.96+1.18) \alpha^{2}\right], }
\end{aligned}
$$

where $\Delta=m_{B}-m_{B^{\prime}}, \alpha=g_{A} / g_{V}$ and $\tau_{B}$ is life time of the parent particle.

Various constraints on the product of $\lambda_{i 1 k}^{\prime} \lambda_{i 2 k}^{\prime}(i=1,2)$ in the units of $\left(m_{\widetilde{d_{k}}} / 100 \mathrm{GeV}\right)^{2}$ from different baryonic decays are listed in the Tables 1 and 2 .

Table 1

\begin{tabular}{|c|c|c|}
\hline Process & BranchingRatio (Expt) & $\lambda_{11 k}^{\prime} \lambda_{12 k}^{\prime}$ \\
\hline \hline$\Lambda \longrightarrow P e^{-} \bar{\nu}_{e}$ & $<(8.32 \pm 0.14) \times 10^{-4}$ & $\begin{array}{c}<1.2 \times 10^{-1}(1 \sigma) \\
<1.3 \times 10^{-1}(2 \sigma)\end{array}$ \\
\hline$\Sigma^{-} \longrightarrow n e^{-} \bar{\nu}_{e}$ & $<(1.017 \pm 0.034) \times 10^{-3}$ & $\begin{array}{r}<8.1 \times 10^{-2}(1 \sigma) \\
<8.5 \times 10^{-2}(2 \sigma)\end{array}$ \\
\hline$\Xi^{-} \longrightarrow \Lambda e^{-} \bar{\nu}_{e}$ & $<(5.63 \pm 0.31) \times 10^{-4}$ & $\begin{array}{l}<1.1 \times 10^{-1}(1 \sigma) \\
<1.2 \times 10^{-1}(2 \sigma)\end{array}$ \\
\hline
\end{tabular}




\section{Table 2}

\begin{tabular}{|c|c|c|}
\hline Process & BranchingRatio(Expt) & $\lambda_{21 k}^{\prime} \lambda_{22 k}^{\prime}$ \\
\hline \hline$\Lambda \longrightarrow P \mu^{-} \bar{\nu}_{\mu}$ & $<(1.57 \pm 0.35) \times 10^{-4}$ & $\begin{array}{l}<1.6 \times 10^{-2}(1 \sigma) \\
<5.3 \times 10^{-3}(2 \sigma)\end{array}$ \\
\hline$\Sigma^{-} \longrightarrow n \mu^{-} \bar{\nu}_{\mu}$ & $<(4.5 \pm 0.4) \times 10^{-4}$ & $\begin{array}{l}<9.6 \times 10^{-3}(1 \sigma) \\
<1.6 \times 10^{-2}(2 \sigma)\end{array}$ \\
\hline$\Xi^{-} \longrightarrow \Lambda \mu^{-} \bar{\nu}_{\mu}$ & $<\left(3.5_{-2.2}^{+3.5}\right) \times 10^{-4}$ & $<5.0 \times 10^{-2}$ \\
\hline
\end{tabular}

Bounds on new set of product couplings summarized in tables 1 and 2, are

within a range obtained for the products $\lambda_{31 k}^{\prime} \lambda_{11 k}^{\prime}, \lambda_{31 k}^{\prime} \lambda_{21 k}^{\prime}$ [12 from the $\tau$ decays into leptons and mesons and $\lambda_{13 k}^{\prime} \lambda_{23 k}^{\prime}, \lambda_{23 k}^{\prime} \lambda_{33 k}^{\prime}, \lambda_{33 k}^{\prime} \lambda_{13 k}^{\prime}$ from the rare decays of $Z$ [13].

In conclusion, we have considered the effects of the SUSY with explicitly broken $R$-parity via $L$-violation on the hypercharge changing semileptonic decays of certain baryons. Interactions induced by $L$-violating operator of the superpotential put constraints on the products of couplings $\lambda_{11 k}^{\prime} \lambda_{12 k}^{\prime}$ and $\lambda_{21 k}^{\prime} \lambda_{22 k}^{\prime}$

\section{References}

[1] H. Nilles, Phys. Rep. 110(1984)1 and references therein; H.E. Haber and G.L. Kane, Phys. Rep. 117(1985)75

[2] V. Barger, G.F. Giudice and T. Han, Phys. Rev. D40(1989)2987

[3] R.N. Mohapatra, Phys. Ret. D34(1986)3457; M.V. KlapdorKleingrothaus and S.G. Kovalendo, Phys. Rev. Lett. 75(1995)17

[4] R.M. Godbole, P. Roy and X. Tata, Nucl. Phys. B401 (1993)67 
[5] K. Agashe and M. Graesser, Phys. Rev. D54(1996)4445; D. Choudhury and P. Roy, Phys. Lett. B378(1996)153

[6] G. Bhattacharyya, J. Ellis and K. Sridhar, Mod. Phys. A10(1995)1583;

G. Bhattacharyya, D. Choudhury and K. Sridhar, Phys. Lett. B355(1995)193

[7] G. Bhattacharyya, preprint IFUP-TH 43/97,hep-ph/9709395

[8] J. Erler, J.L. Feng and N. Polonsky, Phys. Rev. Lett. 78 (1997)3063; D.L. Ghosh, S. Raychaudhuri and K. Sridhar, hep- ph/9608352; D. Choudhury and S. Raychaudhuri, hep-ph/9702392; A. Datta et al., Phys. Rev. D56 (1997)466

[9] F.Zwirner, Phys. Lett. B132(1983)103

[10] C.S. Aulakh and R.N. Mohapatra, Phys. Lett. B 119 (1983) 136; L.J. Hall and M. Suzuki, Nucl. Phys. B231(1984)419; I.H. Lee, Nucl. Phys. B246(1984)120; J. Ellis et al., Phys. Lett. B150 (1985)142; G.G. Ross and J.W. Valle, Phys. Lett. B151(1985)375; S. Dawson, Nucl. Phys. B261(1985)297; R.N. Mohapatra, Phys. Rev. D 34(1986)3457; E. Ma and P. Roy, Phys. Rev. D41(1990)988; E. Ma and D. Ng, Phys. Rev. D41(1990)1005; S. Dimopoulos, R. Esmailzadeh, L.J. Hall, J.P. Merlo and G.S. Starkmen, Phys. Rev. D 41(1990)2099; H. Dreiner and R.J.N. Phillips, Nucl. Phys. B367(1991)591; L.E. Ibàñez and G.G. Ross, Nucl. 
Phys. B368(1992)3; K. Enqvist, A. Masiero and A. Riotto, Nucl. Phys. B373 (1992)95

[11] D. Choudhury and P. Roy, Phys. Lett. 378(1996)153; R. Barbieri, A. Strumia and Z. Berezhiani, hep-ph/9704275

[12] J. Kim, P. Ko, and D.G. Lee, Phys. Rev. D56(1997)100

[13] M. Anwar Mughal, M. Sadiq and K. Ahmed, Phys. Lett. B417 (1998)87

[14] G. Bhattacharyya and A. Raychaudhuri, Phys. Rev. D57(1998)3837 